\title{
Crystal nuclei and structural correlations in two-dimensional colloidal mixtures: experiment versus simulation
}

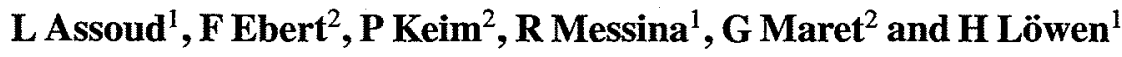 \\ ${ }^{1}$ Institut für Theoretische Physik II: Weiche Materie, Heinrich-Heine-Universität Düsseldorf, \\ Universitätsstraße 1, D-40225 Düsseldorf, Germany \\ ${ }^{2}$ Fachbereich für Physik, Universität Konstanz, D-78457 Konstanz, Germany
}

\begin{abstract}
We examine binary mixtures of superparamagnetic colloidal particles confined to a two-dimensional water-air interface both by real-space experiments and Monte Carlo computer simulations at high coupling strength. In the simulations, the interaction is modelled as a pairwise dipole-dipole repulsion. While the ratio of magnetic dipole moments is fixed, the interaction strength governed by the external magnetic field and the relative composition is varied. Excellent agreement between simulation and experiment is found for the partial pair distribution functions including the fine structure of the neighbour shells at high coupling. Furthermore local crystal nuclei in the melt are identified by bond-orientational order parameters and their contribution to the pair structure is discussed.
\end{abstract}

(Some figures in this article are in colour only in the electronic version)

\section{Introduction}

The mechanisms and principles of heterogeneous crystal nucleation and the subsequent microstructure formation are still far from being explored and understood [1-3]. Steering the nucleation behaviour has important implications relevant for protein crystallization [4] and the formation of new glasses and metallic alloys [5, 6]. Colloidal suspensions have been exploited as model systems for crystal nucleation [7-11]. For instance, it is possible to watch crystal nucleation in real space by using confocal microscopy [12]. In particular, two-dimensional suspensions of superparamagnetic particles confined to the air-water interface of a pending droplet [13] can easily be controlled by an external magnetic field. This allows one to tune the interparticle interactions and to quench the systems quickly into a supercooled state [14]. The external field induces magnetic dipole moments in the particles [15]. If the field direction is normal to the airwater interface, the resulting dipole moments are parallel and the interparticle pair potential is repulsive, scaling with the inverse cube of the particle separation [16]. Binary mixtures of these superparamagnetic colloids are ideal model systems to study crystal nucleation [17], crystallization [18] and glass formation [19] in real space.
In this paper, we consider a binary mixture of superparamagnetic particles both by real-space microscopy experiment and Monte Carlo computer simulations of a binary dipole-dipole interaction model $[20,17]$. In the simulations, the interaction is modelled as a pairwise dipoledipole repulsion. While the ratio of magnetic dipole moments is fixed, the interaction strength governed by the external magnetic field and the relative composition is varied. We compare the pair correlation functions for strong interactions (i.e. for large external magnetic fields) and find good agreement between experiment and simulations. Moreover, we discuss the occurrence of peaks in the distance-resolved pair correlations in conjunction with local crystallites. The latter are patches of triangular and square ordering which are crystalline 'seeds' in the amorphous fluid and building blocks from the globally stable crystalline structure [18]. These crystallites actually could act as nucleation centres for homogeneous nucleation if the system is quenched deeply into the supercooled state [14]. Though heterogeneous nucleation is not tackled in the present paper, an understanding of the local crystallites in the bulk is a first necessary step in order to access possible pathways of inhomogeneous systems leading to heterogeneous nucleation.

As regards previous work, we here address strong couplings different from the weakly coupled case where 
partial clustering of the small particles was found [20] and equilibrated systems. In [14], a quench was performed and the pair correlation functions were found to be widely different from their equilibration counterparts. For this genuinely non-equilibrium phenomenon, Brownian dynamics computer simulations were employed.

This paper is organized as follows: in section 2 we briefly describe the experimental set-up and the simulation method. In section 3, results of experiment and computer simulations are presented and discussed. Finally we conclude in section 4 .

\section{Methods}

\subsection{Experimental system and techniques}

The experimental system consists of a suspension of two kinds of spherical and superparamagnetic colloidal particles. The two species are called A and B, respectively, with B referring to the smaller particles. The hard-core diameters of the two species are $d_{\mathrm{A}}=4.5 \mu \mathrm{m}$ and $d_{\mathrm{B}}=2.8 \mu \mathrm{m}$ and their magnetic susceptibilities are $\chi_{\mathrm{A}}=6.2 \times 10^{-11} \mathrm{~A} \mathrm{~m}^{2} \mathrm{~T}^{-1}$ and $\chi_{\mathrm{B}}=$ $6.6 \times 10^{-12} \mathrm{~A} \mathrm{~m}^{2} \mathrm{~T}^{-1}$. The details of the experimental set-up are explained elsewhere $[17,13,21]$. Due to their high mass density, the particles are confined by gravity to a flat waterair interface formed by a pending water drop. The droplet is suspended by surface tension in a top-sealed cylindrical hole with a diameter of $6 \mathrm{~mm}$ and a depth of $1 \mathrm{~mm}$ in a glass plate. A coil produces a magnetic field $\mathbf{H}$ perpendicular to the water-air interface which induces a magnetic moment (i.e. $\mathbf{m}_{i}=\chi_{i} \mathbf{H}$ with $i=\mathrm{A}, \mathrm{B}$ ) in each particle. This leads to a repulsive dipole-dipole pair interaction [22]. By microscopy, trajectories of all particles in the field of view can be recorded over several days providing sufficient phase space information. The ensemble can be considered as ideally two-dimensional as the thermally activated 'out-of-plane' motion of the particles is in the range of a few nanometres.

While temperature is fixed to room temperature the strength of the interparticle interactions is tunable by the external magnetic field strength. A second parameter which is varied is the relative composition or the mixing ratio of the particles:

$$
X \equiv \begin{gathered}
N_{\mathrm{B}} \\
N_{\mathrm{A}}+N_{\mathrm{B}}
\end{gathered} .
$$

\subsection{Monte Carlo simulation technique}

In our Monte Carlo computer simulations, we model the system in two spatial dimensions by a pairwise additive potential:

$$
u_{i j}(r)=\begin{aligned}
& \mu_{0} \chi_{i} \chi_{j} H^{2} \\
& 4 \pi \quad r^{3}
\end{aligned} \quad(i, j=\mathrm{A}, \mathrm{B})
$$

where $r$ denotes the distance between two particles. For this inverse power potential, at fixed composition $X$, all static quantities depend solely on a dimensionless interaction strength or coupling constant:

$$
\Gamma=\begin{gathered}
\mu_{0} \chi_{\mathrm{A}}^{2} H^{2} \\
4 \pi k_{\mathrm{B}} T a^{3}
\end{gathered}
$$

where $k_{\mathrm{B}} T$ is the thermal energy and $a=1 / \sqrt{ } \rho_{\mathrm{A}}$ is the average interparticle separation between $A$ particles [23]. Hence effective temperature corresponds to the inverse of the coupling $\Gamma$, and the system is completely characterized by three parameters: (1) dipolar moment (or susceptibility) ratio:

$$
m \equiv{ }_{m_{\mathrm{A}}}^{m_{\mathrm{B}}}=\frac{\chi_{\mathrm{B}}}{\chi_{\mathrm{A}}}
$$

(2) the relative composition $X$ and (3) the interaction strength $\Gamma$. While we fix the former to $m=0.1$, the coupling $\Gamma$ and the relative composition $X$ are varied. Standard Monte Carlo simulations were performed with $N_{\mathrm{A}}=400$ A particles and a corresponding number $N_{\mathrm{B}}$ of $\mathrm{B}$ particles determined by the prescribed relative composition $X$. The particles are in a square box with periodic boundary conditions in both directions. Typically $4 \times 10^{6}$ Monte Carlo steps per particle are used for equilibration and statistics is gathered over an additional $10^{6}$ Monte Carlo steps.

\section{Results}

We present our results for the two considered compositions $X=0.29$ and 0.44 . Various coupling strengths $\Gamma$ are then investigated by (i) microstructural analysis and (ii) partial pair distribution functions. In both cases, real-space experiments and Monte Carlo computer simulations have been performed. As a reference, we have gathered in table 1 the different stable crystalline structures at $m=0.1$ from the ground state $(T=0)$ theoretical study [18].

\subsection{Microstructural analysis}

A visual overview of typical microstructures from the experiments are provided in figure 1 . From the theoretical study [18] (see also table 1), it is known that the relevant stable ground state crystals consist of pure A-triangular [T(A)] structures $(X=0)$ and intersecting squares of $\mathrm{A}$ and $\mathrm{B}$ particles [S(AB) phase] at $X=0.5$. Local crystallites in the fluid which possess this order are detected by colouring particles which have a pure triangular and square order ${ }^{3}$

One can clearly see from figure 1 that the triangular $T(A)$ and square phases $S(A B)$ are indeed predominant at strong enough couplings. More precisely, at $X=0.29$, see

3 In detail, we have used criteria to define $A$ particles which have a pure triangular surrounding of other A particles, i.e. which are close to a cut-out of a pure triangular $A$ crystal, and, likewise, we have identified $A$ and $B$ particles which form locally an equimolar square lattice $S(A B)$. The corresponding two structural elements are shown in table 1. In detail, we associate a triangular surrounding to an A particle if the following two criteria are fulfilled simultaneously [17]: (1) the sixfold bond order parameter $p_{6}=\sqrt{ } \Psi_{6}^{*} \Psi_{6}$ (where $\Psi_{6}={ }_{6}^{1} \sum_{\mathrm{NN}}^{6} \exp \left(\mathrm{i} 6 \theta_{\mathrm{NN}}\right)$ with $\theta_{\mathrm{NN}}$ denoting the angles of the six nearest-neighbour bonds relative to a fixed reference) is larger than 0.94 and (2) the relative bond length deviation $b_{6}={ }_{6}^{1} \sum_{\mathrm{NN}}^{6}{ }_{\mathrm{NN}}^{l_{\mathrm{NN}}-\bar{l} \mid}$, where $\bar{l}$ is the average length of the six bond lengths and $l_{\text {NN }}$ is smaller than 0.04 . This double condition selects local configurations close to those of a perfect triangular lattice where $p_{6}$ is unity and $b_{6}$ vanishes. Likewise we define a square surrounding around a $\mathbf{B}$ particle by the criteria: (1) the fourfold bond order parameter $p_{4}=\sqrt{ } \Psi_{4}^{*} \Psi_{4}$ (where $\Psi_{4}={ }_{4}^{1} \sum_{\mathrm{NN}}^{4} \exp \left(i 4 \theta_{\mathrm{NN}}\right)$ with $\theta_{\mathrm{NN}}$ denoting the bond angles of the four nearest-neighbour $\mathrm{AB}$ bonds) is larger than 0.92 and (2) the corresponding relative $\mathrm{AB}$ bond length deviation $b_{4}$ is smaller than 0.05 . 

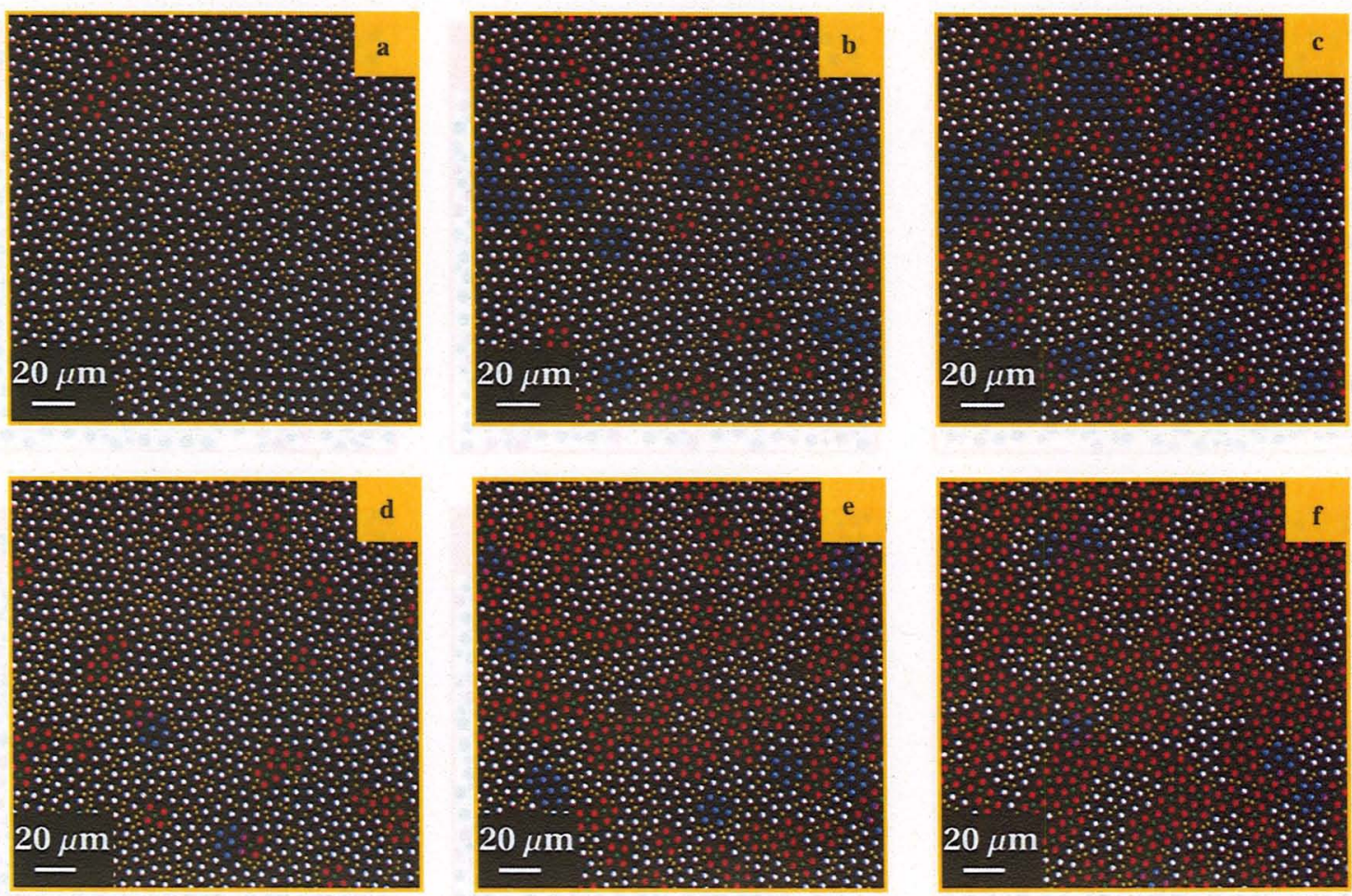

Figure 1. Experimental snapshots for the parameter combinations (a) $X=0.29, \Gamma=4.9$; (b) $X=0.29, \Gamma=38.9$; (c) $X=0.29, \Gamma=82.9$; (d) $X=0.44, \Gamma=22.6$; (e) $X=0.44, \Gamma=49.5$; (f) $X=0.44, \Gamma=93.9$. Large particles are shown in blue if they belong to a triangular surrounding and in red if they belong to a square surrounding. All other large particles are shown in white. A few large particles belonging to both triangular and square surroundings are shown in pink. The small particles are shown in green if they belong to a square centre of large particles, otherwise they appear in yellow.

Table 1. Theoretically predicted stable phases [18] for $m=0.1$ at $T=0$. The same notation as in [18] is used here. The discs (open circles) correspond to A (B) particles.

\begin{tabular}{|c|c|c|c|c|c|c|}
\hline Phase & $\mathrm{T}(\mathrm{A})$ & $\mathbf{R}(\mathrm{A}) \mathrm{A}_{3} \mathrm{~B}$ & $\operatorname{Re}(A) A_{2} B$ & $\mathbf{R}(\mathrm{A}) \mathrm{AB}$ & $\mathrm{R}(\mathrm{A}) \mathrm{A}_{2} \mathrm{~B}_{2}$ & $\mathbf{S}(\mathrm{AB})$ \\
\hline Composition $(X)$ & 0 & $1 / 5$ & $1 / 4$ & $1 / 3$ & $2 / 5$ & $1 / 2$ \\
\hline $\begin{array}{l}\text { Crystalline } \\
\text { structures }\end{array}$ & & & & & $\because \dot{\nabla} \therefore$ & \\
\hline
\end{tabular}

f gures $1(\mathrm{a})-(\mathrm{c})$, there is a strong presence of triangular $\mathrm{T}(\mathrm{A})$ crystallites. This fraction of triangular crystallites is growing with increasing $\Gamma$ : see fi ures $1(\mathrm{a})-(\mathrm{c})$.

At near equimolarity with $X=0.44$, see f gures $1(\mathrm{~d})-(\mathrm{f})$, the situation differs qualitatively (compare with f gures 1 (a)(c)), where we now have a strong fraction of squared $\mathbf{S}(\mathrm{AB})$ crystallites. This fraction is increasing with growing $\Gamma$, which is consistent with the zero-temperature limit predicting the stability of the squared $\mathrm{S}(\mathrm{AB})$ lattice at $X=0.5$ (see table 1 and [18]).

The simulation snapshots are presented in f gure 2 for the same $(\Gamma, X)$ parameters as in fgure 1 . In a general manner, there is an excellent qualitative agreement between the experimental and simulational microstructures, compare f gure 1 with f gure 2 . Very interestingly, the theoretically predicted intermediate rectangular phase $\operatorname{Re}(\mathrm{A}) \mathrm{A}_{2} \mathrm{~B}$, see table 1 for $X=0.25$, is remarkably well presented in the snapshot of fgure 2(c). This feature was not detected in the experiments, compare with $\mathrm{f}$ gure $1(\mathrm{c})$, possibly due to a slightly imperfect equilibration thereby.
The emergence of crystalline clusters in the strongly interacting system gives some insight into the nucleation behaviour. Presumably the system is in a stable crystalline phase at high $\Gamma$ but since it is undercooled it does not f nd the ultimate stable state [24]. The intermittent crystal nucleation 'self-poisons' [25] further crystal growths which may be similar to nucleation in liquid crystalline systems [25].

\subsection{Pair distribution functions}

We now discuss more quantitatively the structural aspects by inspecting the radially averaged partial pair distribution functions, whose corresponding microstructures can be found in f gures 1 and 2 for the experimental and simulational data, respectively.

The case $X=0.29$ is reported in fgure 3 . In a general fashion, there is good quantitative agreement between experiment and simulation, see f gure 3 . The only situation that slightly deviates from this quality of agreement concerns the partial pair distribution $g_{\mathrm{BB}}(r)$ at $\Gamma=82.9$, see f gure $3(\mathrm{c})$. 

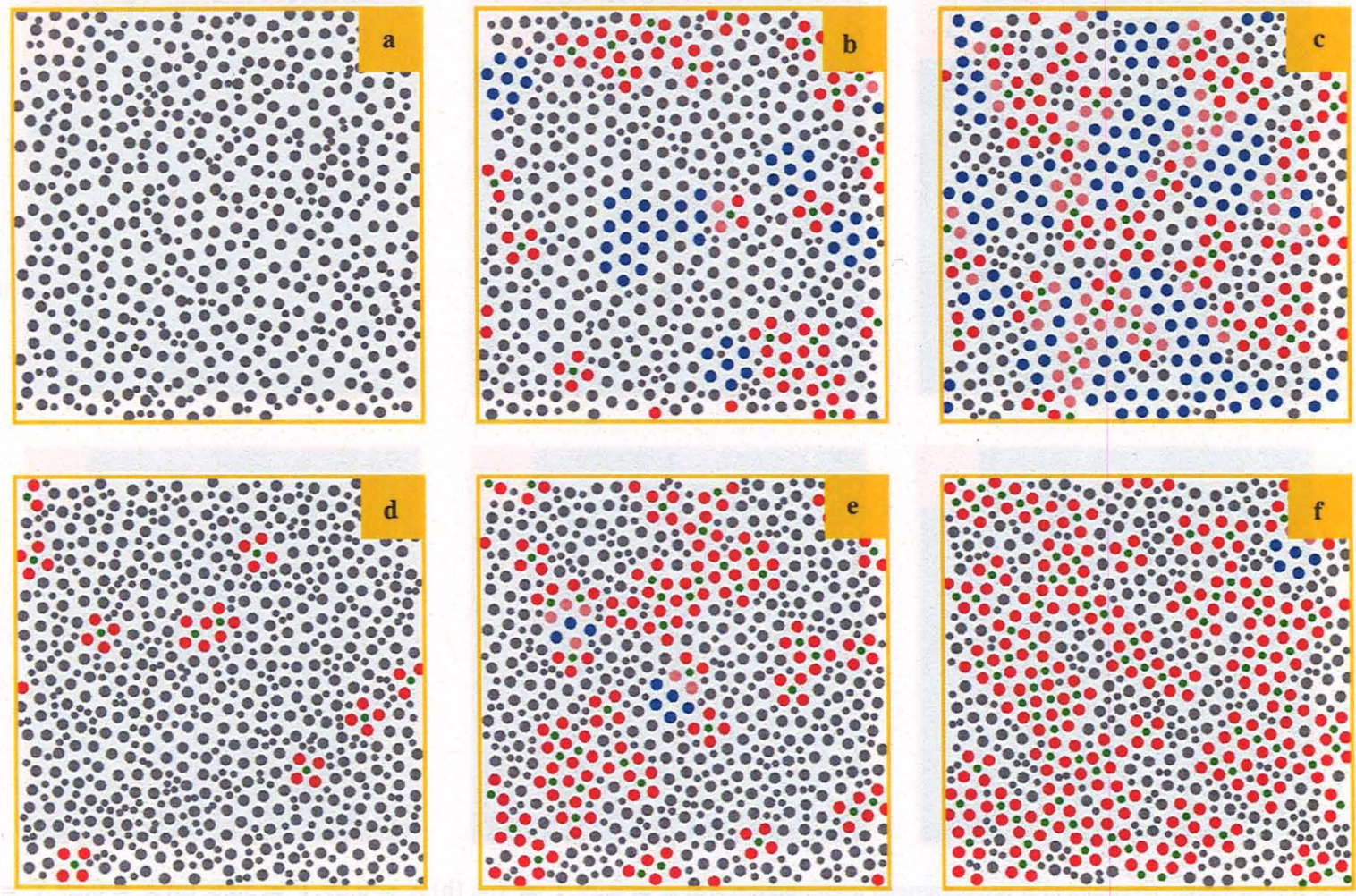

Figure 2. Simulation snapshots for the parameter combinations (a) $X=0.29, \Gamma=4.9$; (b) $X=0.29, \Gamma=38.9$; (c) $X=0.29, \Gamma=82.9$; (d) $X=0.44, \Gamma=22.6$; (e) $X=0.44, \Gamma=49.5$; (f) $X=0.44, \Gamma=93.9$. Large particles are shown in blue if they belong to a triangular surrounding and in red if they belong to a square surrounding. All other large particles are shown in grey. A few large particles belonging to both triangular and square surroundings are shown in pink. The small particles are shown in green if they belong to a square centre of large particles, otherwise they appear in grey.

We explain this with a small drift of the colloidal system in the feld of view. The whole systems contains more than 100000 particles and is susceptible to perturbations. The drift induced some shear which may constrain here the ordering of the system at $\Gamma=82.9$ and $X=0.29$. The primary peaks found at $r / a=1$ in $g_{\mathrm{AA}}(r)$ and at $r / a=1 / \sqrt{ } 2 \approx 0.71$ in $g_{\mathrm{AB}}(r)$ are the signature of the squared $\mathrm{S}(\mathrm{AB})$ crystallites, see f gures 3 (b) and (c).

The disordered aspect of the material can be best identif ed by analysing at the partial pair distribution $g_{\mathrm{BB}}(r)$. Figures $3(\mathrm{~b})$ and (c) show that the frst peak in $g_{\mathrm{BB}}(r)$ is located at $r / a \approx$ 0.45 , which is much smaller than the (square) unit lattice parameter, which is at $r / a=1$. This is reminiscent of the similar trend for the small particles to form pairs and/or clusters at lower coupling [20], as can also be easily observed in the microstructures of $f$ gures 1 and 2 .

The case $X=0.44$ is reported in f gure 4 . The agreement is now even better than at $X=0.29$, becoming perfect for all the partial distribution functions (including $g_{\mathrm{BB}}(r)$ ).

\section{Conclusion}

In conclusion we have put forward the idea that twodimensional binary mixtures are excellent model systems for crystal nucleation as they are easily supercooled by increasing an applied external feld and crystallize into a variety of crystal structures. As revealed by the excellent agreement in the pair correlation functions, the system can be modelled by a simple dipole-dipole interaction potential [22]. The strongly interacting fuid bears some crystallites which were identif ed and could act as possible nucleation centres both for homogeneous and heterogeneous nucleation.

Future investigations should consider the nucleation at fi ed imposed nucleation seeds which was studied theoretically for one-component two-dimensional systems [26]. Steering the nucleation and growth for binary systems is expected to be much richer since there are several competing crystalline structures. Exploring more asymmetries in the magnetic moments is possible by exploiting nonlinear saturation effects in the magnetic susceptibility at high external magnetic f elds. This is another parameter which is crucially determining the phase behaviour. Finally, it might be interesting to use binary charged suspensions conf ned between charged glass plates $[27,28]$ as a two-dimensional model system for crystal nucleation. The interactions are then wellapproximated effective Yukawa potentials $[29,30]$ where the screening length is steered by the salinity. Again the ground state crystal structures show a wealth of possible crystals as recently revealed by lattice-sum calculations [31]. Therefore a rich scenario of crystal nucleation and growth phenomena are expected to occur here as well. Another realization of dipolar mixtures in $2 \mathrm{~d}$ are granular systems [32] 

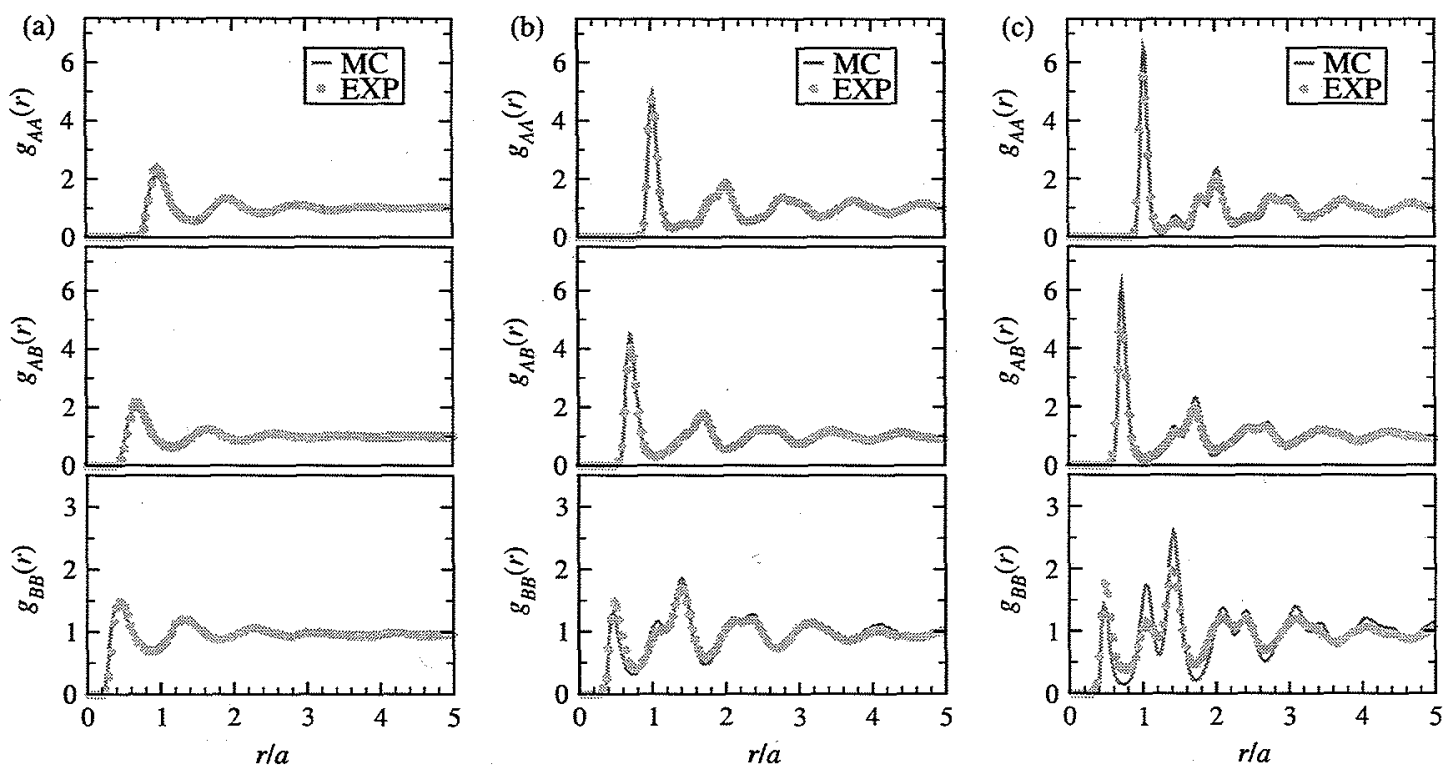

Figure 3. Partial radial pair distribution functions $g_{\mathrm{AA}}(r), g_{\mathrm{BB}}(r)$ and $g_{\mathrm{AB}}(r)$. Experimental data (EXP) are compared to simulation results (MC) for (a) $\Gamma=4.9$, (b) $\Gamma=38.9$ and (c) $\Gamma=82.9$. The composition $X=0.29$ is fixed.
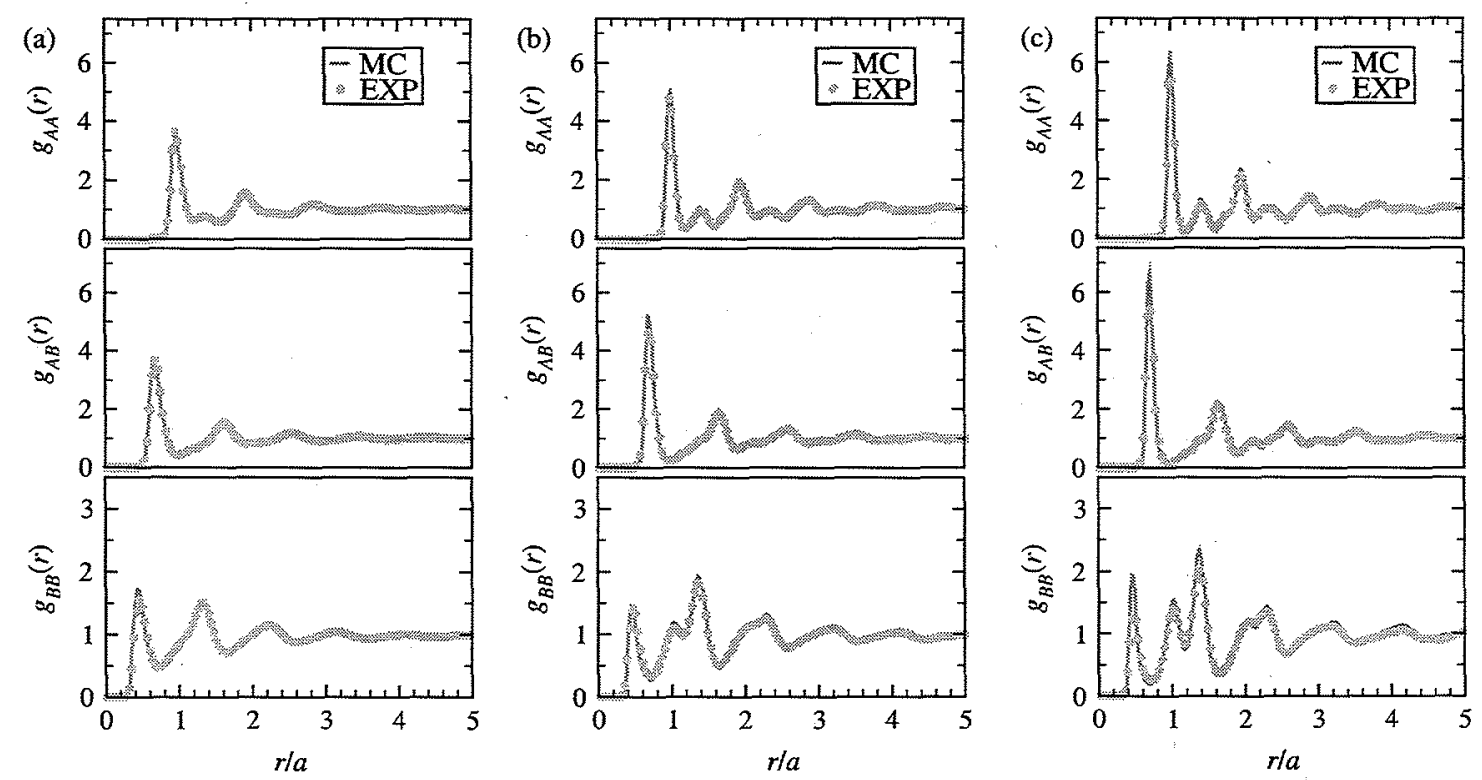

Figure 4. Partial radial pair distribution functions $g_{\mathrm{AA}}(r), g_{\mathrm{BB}}(r)$ and $g_{\mathrm{AB}}(r)$. Experimental data (EXP) are compared to simulation results (MC) for (a) $\Gamma=22.6$, (b) $\Gamma=49.5$ and (c) $\Gamma=93.9$. The composition $X=0.44$ is fixed.

which show interesting $2 d$ ordering effects [33]. Finally binary colloid mixtures with added nonadsorbing polymers will result in effective attractions and possibly liquid-gas phase separation $[34,35]$. The interplay of vitrification or gelation and the fluid-fluid phase separation in two dimensions should be an interesting topic for future research [36].

\section{Acknowledgments}

We thank D R Nelson, Y Terada, and P Dillmann for helpful discussions. This work was supported by the DFG (SPP 1296, SFB-513 project B6 and SFB-TR6 project C2).

\section{References}

[1] Cacciuto A, Auer S and Frenkel D 2004 Nature 428400

[2] Granasy L, Pusztai T, Saylor D and Warren J A 2007 Phys. Rev. Lett. 98035703

[3] Emmerich H 2007 Phil. Mag. Lett. 87795

[4] Saridakis E and Chayen N E 2009 Trends Biotechnol. 2799

[5] Weinberg M C, Neilson G F and Uhlmann D R 2008 J. Non-Cryst. Solids 68115

[6] Emmerich H 2008 Adv. Phys. 571

[7] Schöpe H J and Palberg T 2002 J. Phys.: Condens. Matter 1411573

[8] de Villeneuve V W A, Verboekend D, Dullens R P A Aarts D G A L, Kegel W K and Lekkerkerker H N W 2005 J. Phys.: Condens. Matter $17 \mathrm{~S} 3371$ 
[9] Auer S and Frenkel D 2005 Adv. Polym. Sci. 173149

[10] Wette P, Schöpe H J and Palberg T 2005 J. Chem. Phys. 123174902

[11] Sear.R P 2007 J. Phys.: Condens. Matter 19033101

[12] Gasser U, Weeks E R, Schofield A, Pusey P N and Weitz D A 2001 Science 292258

[13] Keim P, Maret $\mathrm{G}$ and Grünberg $\mathrm{H} \mathrm{H}$ v 2007 Phys. Rev. E 75031402

[14] Assoud L, Ebert F, Keim P, Messina R, Maret G and Löwen H 2009 arXiv:0811.1498v2[Cond-Mat]

[15] Zahn K, Méndez-Alcaraz J M and Maret G 1997 Phys. Rev. Lett. 79175

[16] For a recent work exploiting this model, see e.g. Terada $Y$ and Tokuyama M 2008 AIP Conf. Proc. 982 387-90

[17] Ebert F, Keim P and Maret G 2008 Eur. Phys. J. E 26161

[18] Assoud L, Messina R and Löwen H 2007 Europhys. Lett. 8048001

[19] König H, Hund R, Zahn K and Maret G 2005 Eur. Phys. J. E 18287

[20] Hoffmann N, Ebert F, Likos C N, Löwen H and Maret G 2006 Phys. Rev. Lett. 97078301

[21] Ebert F, Dillmann P, Maret G and Keim P 2009 arXiv:0903.2808[Cond-Mat]

[22] Froltsov V, Blaak R, Likos C N and Löwen H 2003 Phys. Rev. E 68061406
[23] Hansen J-P and MacDonald I R 1986 Theory of Simple Liquids 2nd edn (New York: Academic)

[24] Ebert F, Maret G and Keim P 2009 arXiv:0903.2812[Cond-Mat]

[25] Schilling T and Frenkel D 2004 Phys. Rev. Lett. 92085505

[26] van Teeffelen S, Likos C N and Löwen H 2008 Phys. Rev. Lett. 100108302

[27] Murray C A and Grier D G 1996 Annu. Rev. Phys. Chem. 47421

[28] Lorenz N, Liu J and Palberg T 2008 Colloids Surf. A 319109

[29] Löwen H and Allahyarov E 1998 J. Phys.: Condens. Matter 104147

[30] Allahyarov E, D'Amico I and Löwen $\mathrm{H} 1999$ Phys. Rev. E 603199

[31] Assoud L, Messina R and Löwen H $2008 \mathrm{~J}$. Chem. Phys. 129164511

[32] Hay M B, Workman R K and Manne S 2003 Phys. Rev. E 67012401

[33] Nelson D R, Rubinstein M and Spaepen F 1982 Phil. Mag. A 46105

[34] Louis A A, Bolhuis P G, Meijer E J and Hansen J P 2002 J. Chem. Phys. 1171893

[35] Rotenberg B, Dzubiella J, Hansen J P and Louis A A $2004 \mathrm{Mol}$. Phys. 1021

[36] Zaccarelli E, Lu P J, Ciulla F, Weitz D A and Sciortino F 2008 J. Phys.: Condens. Matter 20494242 